\title{
Heavy flavour production in $p p$ collisions and intrinsic quark components in proton
}

\section{G. I. Lykasov*}

Joint Institute for Nuclear Research

E-mail: lykasov@jinr.ru

\section{A. A. Grinyuk}

Joint Institute for Nuclear Research

E-mail: andrei.grinyuk@gmail.com

\section{V. Bednyakov}

Joint Institute for Nuclear Research

E-mail: bednyakovi@gmail.com

The LHC data on the forward heavy flavour hadron production can be a new unique source for estimation of intrinsic heavy quark contributions to the proton. We discuss in detail the $D$-meson production in $p p$ collisions at the LHC including the intrinsic charm in the proton. We present also some predictions for the $K$-meson production in the $p p$ collision at the initial energies $158 \mathrm{GeV}$ and $7 \mathrm{TeV}$ made within the perturbative QCD including the intrinsic strangeness in the proton that can be verified in the NA61 experiment and at LHC.

XXI International Baldin Seminar on High Energy Physics Problems

September 10 -15, 2012

JINR, Dubna, Russia

* Speaker. 


\section{Intrinsic heavy flavours in the proton}

The Large Hadron Collider (LHC) at CERN can be a useful laboratory for investigation of the unique structure of the proton, in particular for the study of the parton distribution functions (PDFs) with high accuracy. It is well known that the precise knowledge of these PDFs is very important for verification of the Standard Model and search for New Physics.

By definition, the PDF $f_{a}(x, \mu)$ is a function of the proton momentum fraction $x$ carried by parton $a$ (quark $q$ or gluon $g$ ) at the QCD momentum transfer scale $\mu$. For small values of $\mu$, corresponding to the long distance scales less than $1 / \mu_{0}$, the PDF cannot be calculated from the first principles of QCD (although some progress in this direction has been recently achieved within the lattice methods [1]). The PDF $f_{a}(x, \mu)$ at $\mu>\mu_{0}$ can be calculated by solving the perturbative QCD evolution equations (DGLAP) [2]. The unknown (input for the evolution) functions $f_{a}\left(x, \mu_{0}\right)$ can usually be found empirically from some "QCD global analysis" [3, 4] of a large variety of data, typically at $\mu>\mu_{0}$.

In general, almost all $p p$ processes that took place at the LHC energies, including the Higgs boson production, are sensitive to the charm $f_{c}(x, \mu)$ or bottom $f_{b}(x, \mu)$ PDFs. Nevertheless, within the global analysis the charm content of the proton at $\mu \sim \mu_{c}$ and the bottom one at $\mu \sim \mu_{b}$ are both assumed to be negligible. Here $\mu_{c}$ and $\mu_{b}$ are typical energy scales relevant to the $c$ - and $b$-quark QCD excitation in the proton. These heavy quark components arise in the proton only perturbatively with increasing $Q^{2}$-scale through the gluon splitting in the DGLAP $Q^{2}$ evolution [2]. Direct measurement of the open charm and open bottom production in the deep inelastic processes (DIS) confirms the perturbative origin of heavy quark flavours [5]. However, the description of these experimental data is not sensitive to the heavy quark distributions at relatively large $x(x>$ $0.1)$.

As was assumed by Brodsky with coauthors in $[6,7]$, there are extrinsic and intrinsic contributions to the quark-gluon structure of the proton. Extrinsic (or ordinary) quarks and gluons are generated on a short time scale associated with a large-transverse-momentum processes. Their distribution functions satisfy the standard QCD evolution equations. Intrinsic quarks and gluons exist over a time scale which is independent of any probe momentum transfer. They can be associated with bound-state (zero-momentum transfer regime) hadron dynamics and are believed to be of nonperturarbative origin. Figure 1 gives a schematic view of a nucleon, which consists of three valence quarks $\mathrm{q}_{\mathrm{v}}$, quark-antiquark $\mathrm{q} \overline{\mathrm{q}}$ and gluon sea, and, for example, pairs of the intrinsic charm $\left(\mathrm{q}_{\text {in }}^{\mathrm{c}} \overline{\mathrm{q}}_{\mathrm{in}}^{\mathrm{c}}\right)$ and intrinsic bottom quarks $\left(\mathrm{q}_{\mathrm{in}}^{\mathrm{b}} \overline{\mathrm{q}}_{\mathrm{in}}^{\mathrm{b}}\right)$.

It was shown in [7] that the existence of intrinsic heavy quark pairs $c \bar{c}$ and $b \bar{b}$ within the proton state could be due to the virtue of gluon-exchange and vacuum-polarization graphs. On this basis, within the MIT bag model [8], the probability to find the five-quark component $|u u d c \bar{c}\rangle$ bound within the nucleon bag was estimated to be about $1-2 \%$.

Initially in $[6,7]$ S.Brodsky with coauthors have proposed existence of the 5-quark state $|u u d c \bar{c}\rangle$ in the proton (Fig. 1). Later some other models were developed. One of them considered a quasi-two-body state $\bar{D}^{0}(u \bar{c}) \bar{\Lambda}_{c}^{+}(u d c)$ in the proton [9]. In [9]-[11] the probability to find the intrinsic charm (IC) in the proton (the weight of the relevant Fock state in the proton) was assumed to be $1-3.5 \%$. The probability of the intrinsic bottom (IB) in the proton is suppressed by the factor $m_{c}^{2} / m_{b}^{2} \simeq 0.1$ [12], where $m_{c}$ and $m_{b}$ are the masses of the charmed and bottom quarks. 


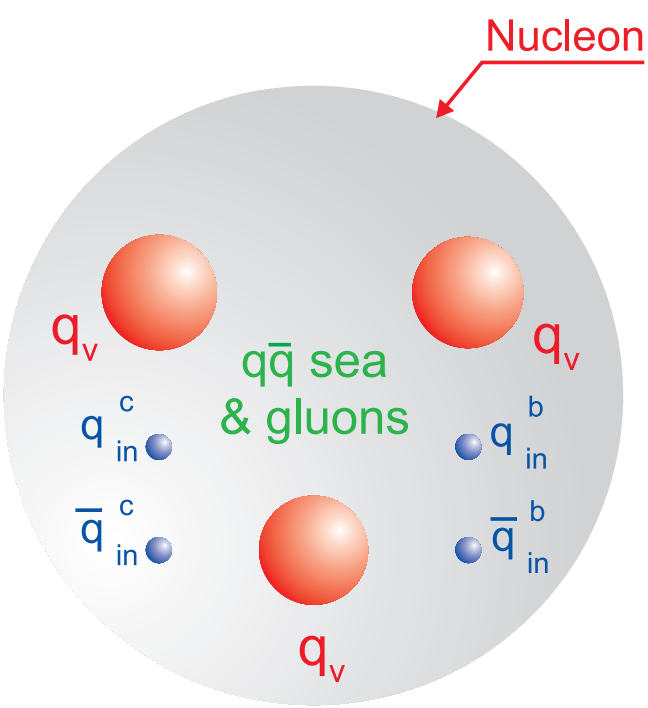

Figure 1: Schematic presentation of a nucleon consisting of three valence quarks $\mathrm{q}_{\mathrm{v}}$, quark-antiquark $q \overline{\mathrm{q}}$ and gluon sea, and pairs of the intrinsic charm $\left(\mathrm{q}_{\text {in }}^{\mathrm{c}} \overline{\mathrm{q}}_{\text {in }}^{\mathrm{c}}\right)$ and intrinsic bottom quarks $\left(\mathrm{q}_{\text {in }}^{\mathrm{b}} \overline{\mathrm{q}}_{\text {in }}^{\mathrm{b}}\right)$.

Nevertheless, it was shown that the IC could result in a sizable contribution to the forward charmed meson production [13]. Furthermore the IC "signal" can constitute almost $100 \%$ of the inclusive spectrum of $D$-mesons produced at high pseudorapidities $\eta$ and large transverse momenta $p_{T}$ in $p p$ collisions at LHC energies [14].

If the distributions of the intrinsic charm or bottom in the proton are hard enough and are similar in the shape to the valence quark distributions (have the valence-like form), then the production of the charmed (bottom) mesons or charmed (bottom) baryons in the fragmentation region should be similar to the production of pions or nucleons. However, the yield of this production depends on the probability to find the intrinsic charm or bottom in the proton, but this yield looks too small. The PDF which included the IC contribution in the proton have already been used in the perturbative QCD calculations in [9]-[11].

The probability distribution for the 5-quark state $(u u d c \bar{c})$ in the light-cone description of the proton was first calculated in [6]. Assuming that the light quark $(u, d)$ masses and the proton mass are smaller than the $c$-quark mass one can get the following form for this probability [10] at $Q^{2}=m_{c}^{2}\left(m_{c}=1.69\right.$ is the c-quark mass):

$$
\frac{d P}{d x}=f_{c}(x)=f_{\bar{c}}(x)=\mathscr{N} x^{2}\left\{(1-x)\left(1+10 x+x^{2}\right)+6 x(1+x) \ln (x)\right\},
$$

where the normalization constant $\mathscr{N}$ determines some probability $w_{\text {IC }}$ to find the Fock state $|u u d c \bar{c}\rangle$ in the proton. The solid line in Fig. 2 shows the intrinsic charm PDF $x f_{c}(x)$ as a function of $x$ at $Q^{2}=m_{c}^{2}$ when the probability $w_{\text {IC }}=3.5 \%$. The dashed curve in Fig. 2 is the density distribution of the (ordinary) sea charm in the proton. One can see from Fig. 2 that the IC distribution (with $w_{\mathrm{IC}}=3.5 \%$ [11]) given by Eq. (1.1) has rather visible enhancement at $x \sim 0.2-0.3$ and it is much larger (a few orders of magnitude) than the sea (ordinary) charm density distribution in the proton. The valence quark density distributions $\left(x f_{u . d}\right)$, the distributions of the sea charm $\left(c_{\text {sea }}\right)$ and the intrinsic charm $\left(x f_{\mathrm{IC}}(x)\right)$ at $Q^{2}=100 \mathrm{GeV} / c^{2}$ are presented in Fig. 3. One can see from Fig. 3. that 


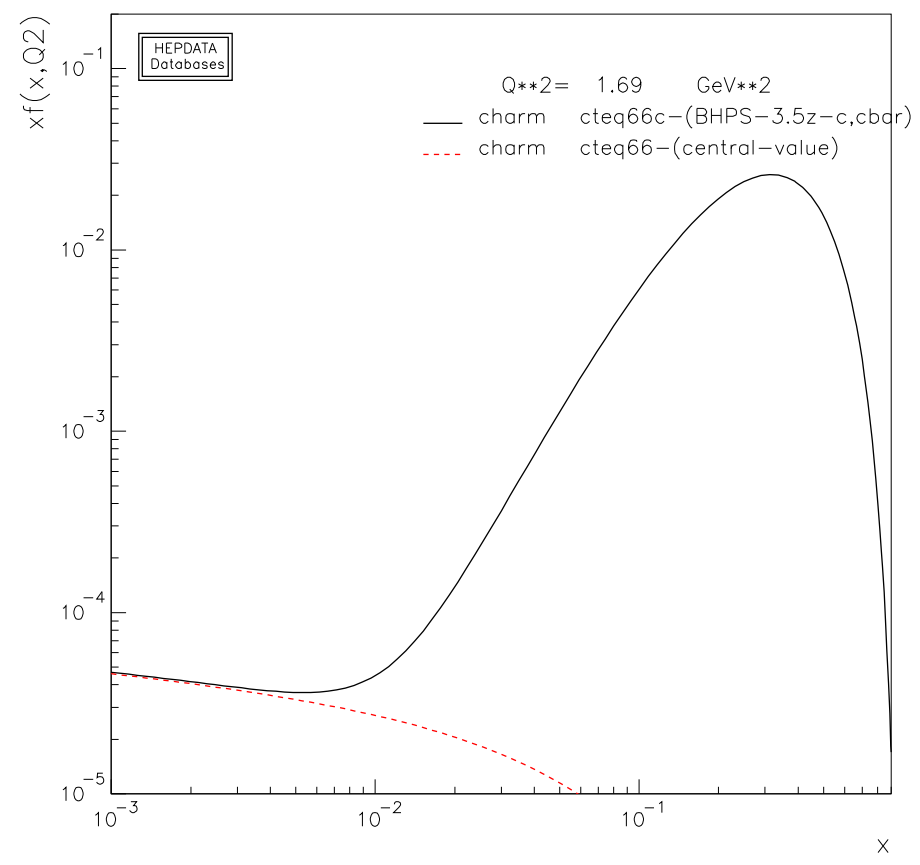

Figure 2: The distributions of charmed quarks in the proton; the dashed line is the sea charmed quarks $c(x)$, the solid curve is the sum of the intrinsic charm ans the sea one $c(x)+c_{\text {in }}(x)$

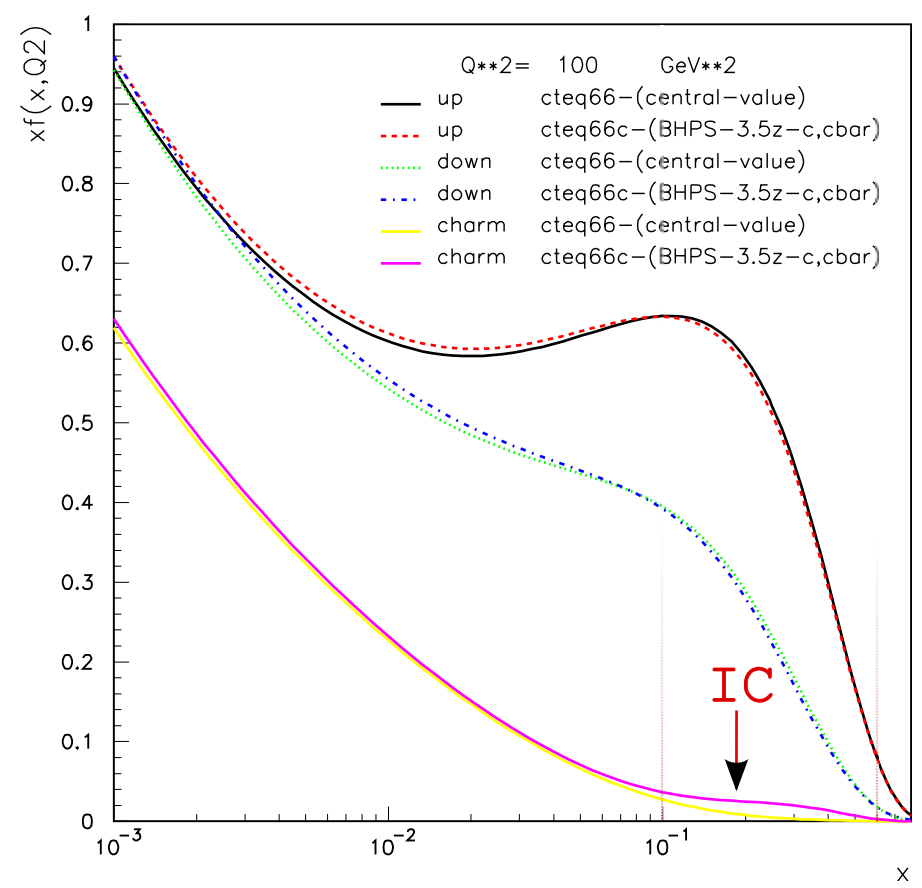

Figure 3: The distributions of the valence $(u, d)$ quarks and sea quarks in the proton as a function of the Bjorken variable $x$. 
the sea charm and the intrinsic charm distributions are much "smaller" than the valence ones in the whole region of $x$. However, in the hard $p p$ collisions the gluons and sea quarks make the main contribution to the inclusive hadron spectra. Therefore, the inclusion of the intrinsic heavy quark components in the proton makes sense.

Due to the nonperturbative intrinsic heavy quark components one can expect some excess of the heavy quark PDFs over the ordinary sea quark PDFs at $x>0.1$. The "signal" of these components can be visible in the observables of the heavy flavour production in semi-inclusive $e p$ DIS and inclusive $p p$ collisions at high energies. For example, it was recently shown that rather good description of the HERMES data on the $x f_{s}\left(x, Q^{2}\right)+x f_{\bar{s}}\left(x, Q^{2}\right)$ at $x>0.1$ and $Q^{2}=$ $2.5 \mathrm{GeV} / c^{2}[15]$ could be achieved due to existence of intrinsic strangeness in the proton. Similarly, possible existence of the intrinsic charm in the proton can lead to some enhancement in the inclusive spectra of the open charm hadrons, in particular $D$-mesons, produced at the LHC in $p p$-collisions at high pseudorapidities $\eta$ and large transverse momenta $p_{T}$ [14].

\section{Intrinsic heavy quarks at the LHC}

\section{- Intrinsic charm}

It is known that in the open charm/beauty $p p$-production at large momentum transfer the hard QCD interactions of two sea quarks, two gluons and a gluon with a sea quark play the main role. According to the model of hard scattering [21]-[29] the relativistic invariant inclusive spectrum of the hard process $p+p \rightarrow h+X$ can be related to the elastic parton-parton subprocess $i+j \rightarrow i^{\prime}+j^{\prime}$, where $i, j$ are the partons (quarks and gluons). This spectrum can be presented in the following general form [25]-[27] (see also [30, 31]):

$$
E \frac{d \sigma}{d^{3} p}=\sum_{i, j} \int d^{2} k_{i T} \int d^{2} k_{j T} \int_{x_{i}^{\min }}^{1} d x_{i} \int_{x_{j}^{\min }}^{1} d x_{j} f_{i}\left(x_{i}, k_{i T}\right) f_{j}\left(x_{j}, k_{j T}\right) \frac{d \sigma_{i j}(\hat{s}, \hat{t})}{d \hat{t}} \frac{D_{i, j}^{h}\left(z_{h}\right)}{\pi z_{h}} .
$$

Here $k_{i, j}$ and $k_{i, j}^{\prime}$ are the four-momenta of the partons $i$ or $j$ before and after the elastic parton-parton scattering, respectively; $k_{i T}, k_{j T}$ are the transverse momenta of the partons $i$ and $j$; $z$ is the fraction of the hadron momentum from the parton momentum; $f_{i, j}$ is the PDF; and $D_{i, j}$ is the fragmentation function (FF) of the parton $i$ or $j$ into a hadron $h$.

When the transverse momenta of the partons are neglected in comparison with the longitudinal momenta, the variables $\hat{s}, \hat{t}, \hat{u}$ and $z_{h}$ can be presented in the following forms [25]:

$$
\hat{s}=x_{i} x_{j} s, \quad \hat{t}=x_{i} \frac{t}{z_{h}}, \quad \hat{u}=x_{j} \frac{u}{z_{h}}, \quad z_{h}=\frac{x_{1}}{x_{i}}+\frac{x_{2}}{x_{j}},
$$

where

$$
x_{1}=-\frac{u}{s}=\frac{x_{T}}{2} \cot (\theta / 2), \quad x_{2}=-\frac{t}{s}=\frac{x_{T}}{2} \tan (\theta / 2), \quad x_{T}=2 \sqrt{t u} / s=2 p_{T} / \sqrt{s} .
$$

Here as usual, $s=\left(p_{1}+p_{2}\right)^{2}, t=\left(p_{1}-p_{1}^{\prime}\right)^{2}, u=\left(p_{2}-p_{1}^{\prime}\right)^{2}$, and $p_{1}, p_{2}, p_{1}^{\prime}$ are the 4-momenta of the colliding protons and the produced hadron $h$, respectively; $\theta$ is the scattering angle for the hadron $h$ in the $p p$ c.m.s. The lower limits of the integration in (2.1) are

$$
x_{i}^{\min }=\frac{x_{T} \cot \left(\frac{\theta}{2}\right)}{2-x_{T} \tan \left(\frac{\theta}{2}\right)}, \quad x_{j}^{\min }=\frac{x_{i} x_{T} \tan \left(\frac{\theta}{2}\right)}{2 x_{i}-x_{T} \cot \left(\frac{\theta}{2}\right)} .
$$


Actually, the parton distribution functions $f_{i}\left(x_{i}, k_{i T}\right)$ also depend on the four-momentum transfer squared $Q^{2}$ that is related to the Mandelstam variables $\hat{s}, \hat{t}, \hat{u}$ for the elastic parton-parton scattering [27]

$$
Q^{2}=\frac{2 \hat{s} \hat{t} \hat{u}}{\hat{s}^{2}+\hat{t}^{2}+\hat{u}^{2}}
$$

Calculating spectra by Eq.(2.1) we used the PDF which includes the IS (and does not include it) [11], the FF of the type AKK08 [29] and $d \sigma_{i j}(\hat{s}, \hat{t}) / d \hat{t}$ calculated within the LO QCD and presented, for example, in [28].

In particular, Fig. 4 shows our estimation of the inclusive yield of single $D^{0}$-mesons in $p p$ production at $\sqrt{s}=7 \mathrm{TeV}$ and $10 \mathrm{GeV} / c<p_{T}<25 \mathrm{GeV} / c$ as a function of the pseudorapidity $\eta$. This estimation is obtained within PYTHIA8, where the PDF CTEQ66 (without the IC, given by

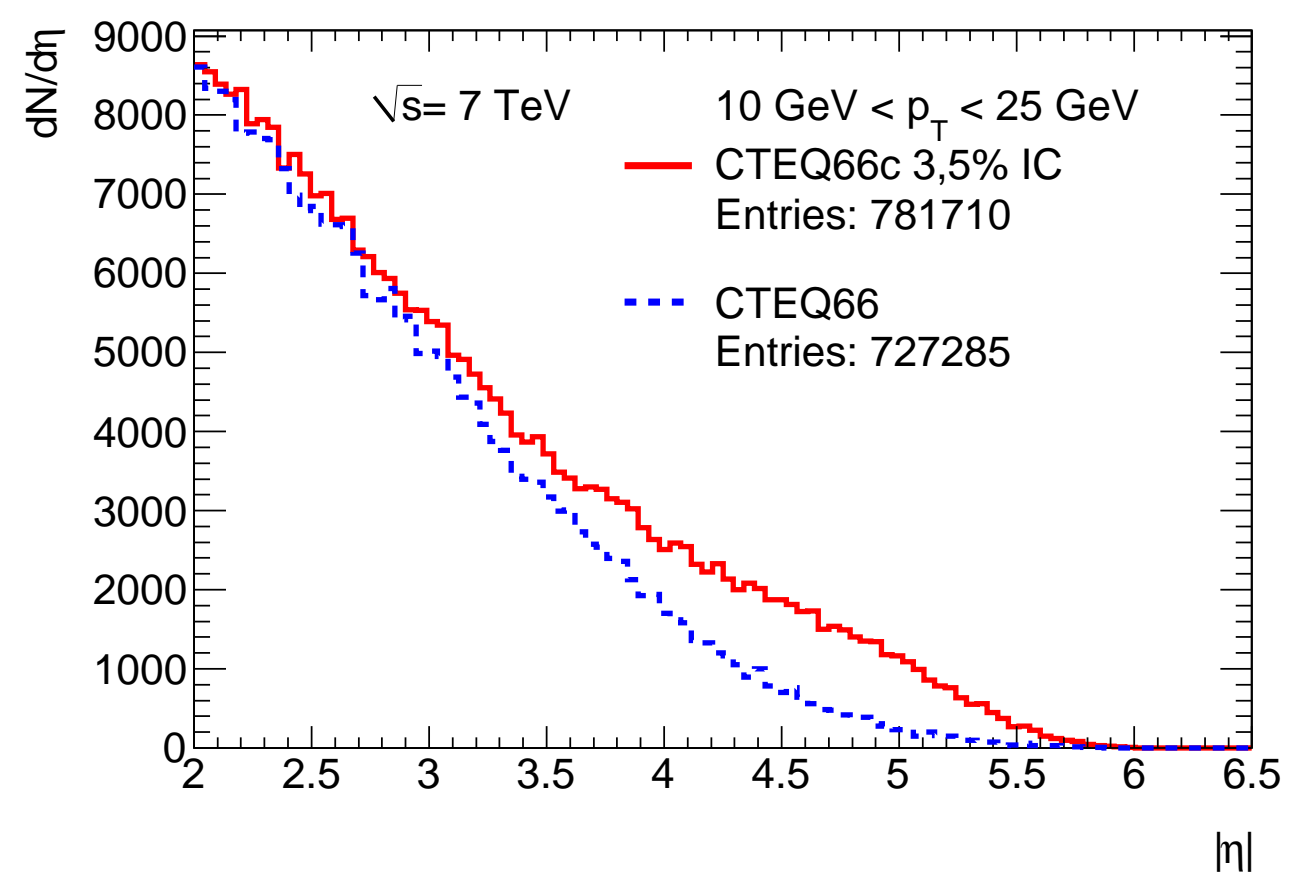

Figure 4: The $D+\bar{D}_{0}$ distributions (with and without intrinsic charm contribution) over the pseudorapidity $\eta$ for $p p \rightarrow\left(D_{0}+\bar{D}_{0}\right)+X$ at $\sqrt{s}=7 \mathrm{TeV}$ and $10 \leq p_{T} \leq 25 \mathrm{GeV} / c$ [14].

the dashed blue curve in Fig. 4) and CTEQ66c (with the IC for $w_{\mathrm{IC}} \simeq 3.5 \%$, given by the solid red curve) were used at $Q^{2}=m_{c}^{2}=(1.69 \mathrm{GeV})^{2}$ [11].

One can see from Fig. 4 that there in some enhancement due to the IC contribution at large $\eta$, which is due to the above-mentioned enhancement in the IC PDF at $x>0.1$, given in Fig. 2. Its amount increases with growing $p_{T}$. For example, due to the IC the spectrum increases by a factor of 2 at $\eta=4.5$. A similar effect was predicted in [32].

One can see that the Feynman variable $x_{F}$ of the produced hadron, for example, the $D^{0}$-meson, can be expressed via the variables $p_{T}$ and $\eta$, or $\theta$ the hadron scattering angle in the $p p$ c.m.s,

$$
x_{F} \equiv \frac{2 p_{z}}{\sqrt{s}}=\frac{2 p_{T}}{\sqrt{s}} \frac{1}{\tan \theta}=\frac{2 p_{T}}{\sqrt{s}} \sinh (\eta) .
$$


At small scattering angles of the produced hadron this formula becomes

$$
x_{F} \sim \frac{2 p_{T}}{\sqrt{s}} \frac{1}{\theta} .
$$

It is clear that for fixed $p_{T}$ an outgoing hadron must possess a very small $\theta$ or very large $\eta$ in order to have large $x_{F}$ (to follow forward, or backward direction).

In the fragmentation region (of large $x_{F}$ ) the Feynman variable $x_{F}$ of the produced hadron is related to the variable $x$ of the intrinsic charm quark in the proton, and according to the longitudinal momentum conservation law, the $x_{F} \simeq x$ (and $x_{F}<x$ ). Therefore, the visible excess of the solid (red) histogram over the dashed (blue) one in Fig. 4 at $\eta>3.5$ is due to the enhancement of the IC distribution (see Fig. 2) at $x>0.1$.

One expects similar enhancement in the experimental distributions of the open bottom production due to the (hidden) intrinsic bottom (IB) in the proton, which could have the PDF very similar to the one given in (1.1). However, the probability $w_{\mathrm{IB}}$ to find in the proton the Fock state with the IB contribution $|u u d b \bar{b}\rangle$ is about 10 times lower than the IC probability $w_{\text {IC }}$ due to the relation $w_{\mathrm{IB}} / w_{\mathrm{IC}} \sim m_{c}^{2} / m_{b}^{2}$, where $m_{b}$ is the bottom quark mass [7, 12].

\section{- Intrinsic strangeness}

Let us analyze now how the possible existence of the intrinsic strangeness in the proton can be visible in $p p$ collisions. For example, consider the $K^{-}$-meson production in the process $p p \rightarrow$ $K^{-}+X$. Considering the intrinsic strangeness in the proton [15] we calculated the inclusive spectrum $E D \sigma / d^{3} p$ of such mesons within the hard scattering model (Eq.(2.1)), which describes satisfactorily the HERA and HERMES data on the DIS. The FF and the parton cross sections were taken from $[29,28]$, respectively, as mentioned above. In Figs. $(5,6)$ the inclusive $p_{t}$-spectra of

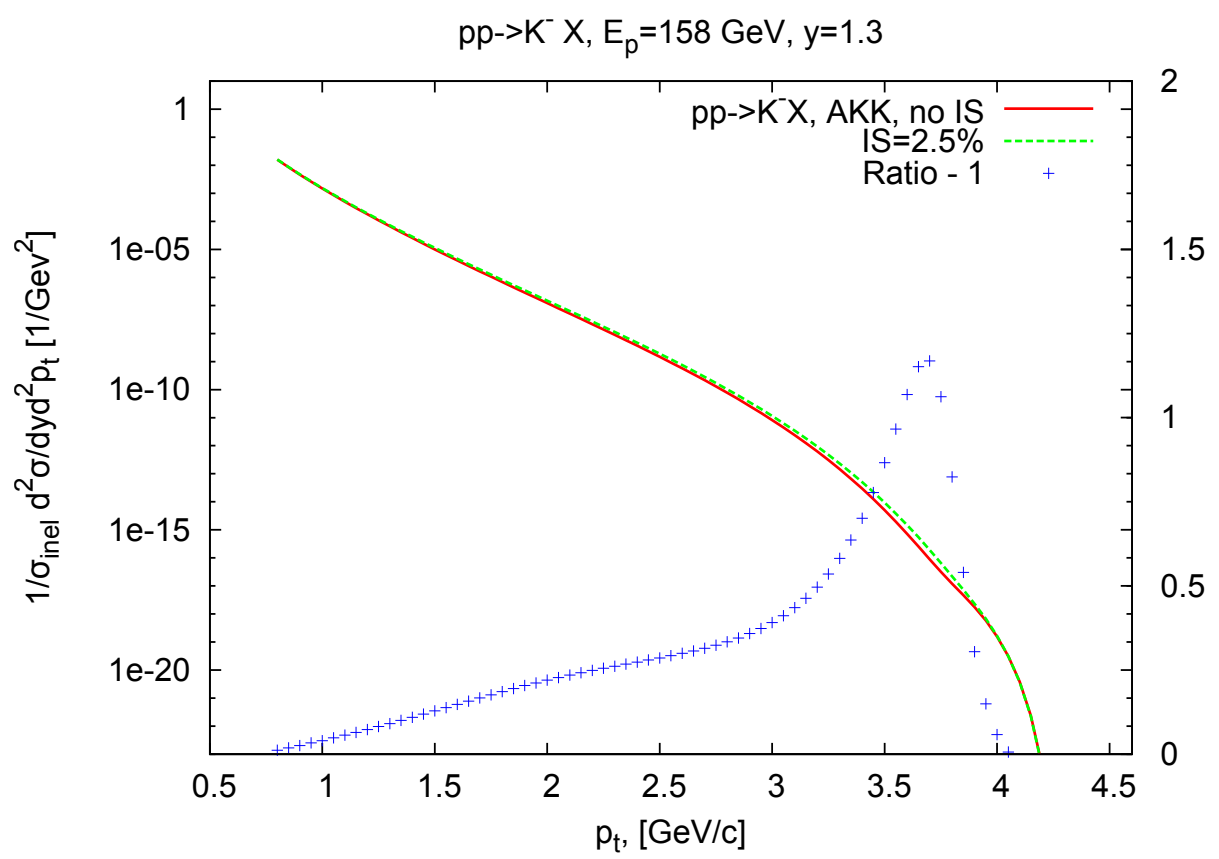

Figure 5: The $K^{-}$-meson distributions (with and without intrinsic charm contribution) over the transverse momentum $p_{t}$ for $p p \rightarrow K^{-}+X$ at the initial energy $E=158 \mathrm{GeV}$, the rapidity $y=1.3$ and $p_{t} \geq 0.8 \mathrm{GeV} / \mathrm{c}$. 


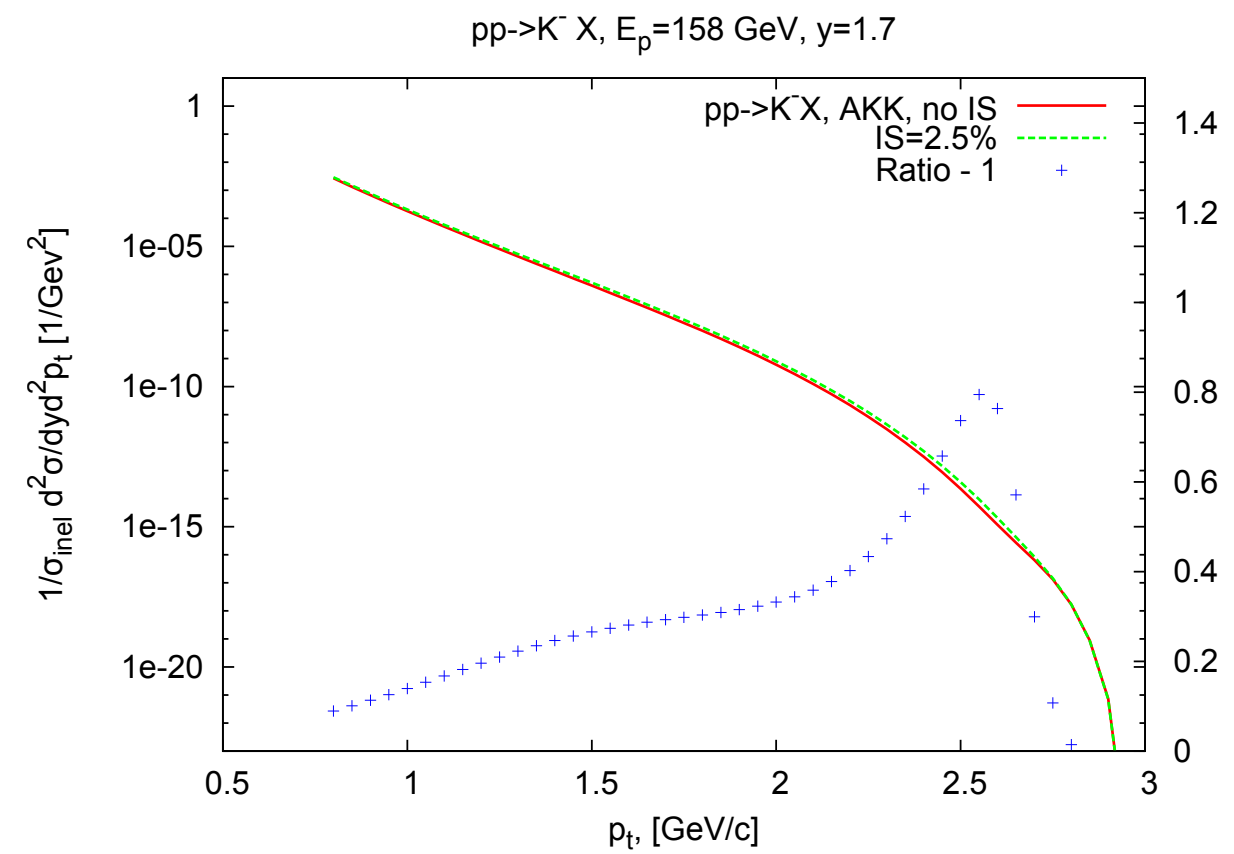

Figure 6: The $K^{-}$-meson distributions (with and without intrinsic charm contribution) over the transverse momentum $p_{t}$ for $p p \rightarrow K^{-}+X$ at the initial energy $E_{p}=158 \mathrm{GeV}$, the rapidity $y=1.7$ and $p_{t} \geq 0.8 \mathrm{GeV} / \mathrm{c}$.

$K^{-}$-mesons produced in $p p$ collision at the initial energy $E_{p}=158 \mathrm{GeV}$ are presented at the rapidity $y=1.3$ (Fig 5) and $y=1.7$ (Fig 6). The solid lines in Figs. $(5,6)$ correspond to our calculation ignoring the intrinsic strangeness (IS) in the proton and the dashed curves correspond to the calculation including the IS with the probability about $2.5 \%$, according to [15]. The crosses show the ratio of our calculation with the IS and without the IS minus 1. One can see from Figs. (5,6, right axis) that the IS signal can be above $200 \%$ at $y=1.3, p_{t}=3.6-3.7 \mathrm{Gev} / \mathrm{c}$ and slightly smaller, than $200 \%$ at $y=1.7, p_{t} \simeq 2.5 \mathrm{Gev} / \mathrm{c}$. Actually, this is our prediction for the NA61 experiment that is now under way at CERN.

We also calculated the inclusive spectra of $K^{-}$and $K^{+}$mesons produced in the $p p$ collision at LHC energies. Note that the spectra of $K^{-}$-mesons, which consist of $s$ - and $\bar{u}$ - quarks, can give us information on the IS at large $x_{F}\left(x_{F}>1\right)$ or at the certain region of $p_{T}$ and $\eta$, according to Eq.(2.6) and Fig. (2), because they are produced mainly from the fragmentation of the strange sea $\left(s_{\text {sea }}\right)$ and the intrinsic strange $\left(s_{i n}\right)$ quarks in the proton. One can see from Fig. (7, right axis) that the IS signal at $\sqrt{s}=7 \mathrm{TeV}, y=4.5$ can be about $400 \%$ at $p_{T} \simeq 32-33 \mathrm{GeV} / \mathrm{c}$, the measuring of which at LHC can be difficult. Anyway, at $p_{T}>15 \mathrm{GeV} / \mathrm{c}$ and $y=4.5$ the IS signal is about $200 \%$ and more, as the crosses show in Fig. (7,right axis). However, the spectra of $K^{+}$-mesons almost do not give us such information because $K^{+}$-meson consists of the $u$ - and $\bar{s}$-quarks and its production at large $x_{F}$ is due to the fragmentation of the valence $u$-quark by the $p p$ collision. It illustrates Fig. (8), the notations are the same as in Figs. $(5,6)$. The similar effect but for the $I C$ signal can be visible in the inclusive spectra of $D^{-}$- and $D^{+}$-mesons produced in $p p$ collision at LHC energies.

Let us also note that we calculated the inclusive spectra of $K$ - and $D$-mesons within the LO QCD. The NLO calculation, in principle, can change the inclusive spectra. However, the ratio of 
the spectrum of $K^{-}$(or $D^{-}$) to the spectrum of $K^{+}$(or $D^{+}$) can not be very sensitive to the NLO corrections.

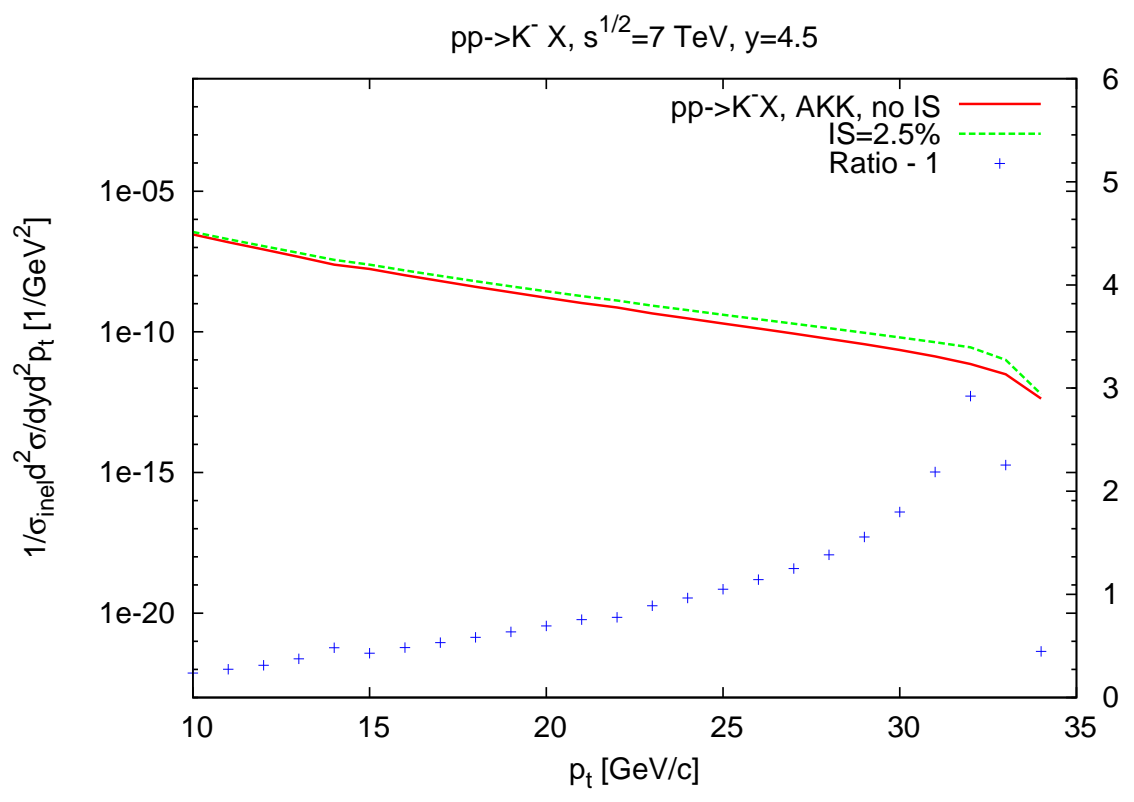

Figure 7: The $K^{-}$-meson distributions (with and without intrinsic charm contribution) over the transverse momentum $p_{t}$ for $p p \rightarrow K^{-}+X$ at $\sqrt{s}=7 \mathrm{TeV}$, the rapidity $y=4.5$ and $p_{t} \geq 10 \mathrm{GeV} / \mathrm{c}$.

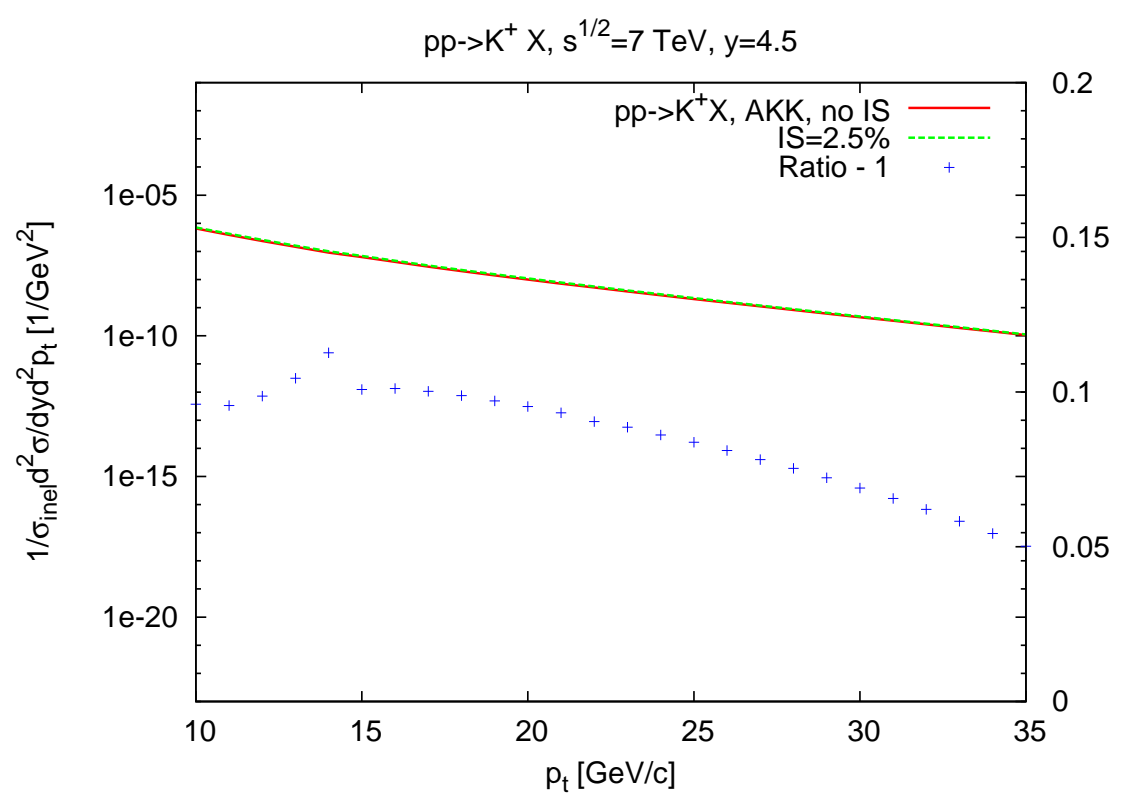

Figure 8: The $K^{+}$-meson distributions (with and without intrinsic charm contribution) over the transverse momentum $p_{t}$ for $p p \rightarrow K^{+}+X$ at $\sqrt{s}=7 \mathrm{TeV}$, the rapidity $y=4.5$ and $p_{t} \geq 10 \mathrm{GeV} / \mathrm{c}$. 


\section{Conclusion}

In this paper we have shown that possible existence of the intrinsic heavy flavour quark components in the proton can be seen by the forward production of the open heavy flavour in $p p$-collisions.

Our calculations of the charmed meson production in $p p$ collisions within the hard scattering model using the PYTHIA8 MC generator [14] and the PDF including the intrinsic charm [11] showed the following. We found that the contribution of the intrinsic charm in the proton could be studied in the production of $D$-mesons in $p p$ collisions at the LHC. The IC contribution for the single $D^{0}$-meson production can be large, about $100 \%$ at high rapidities $3 \leq y \leq 4.5$ and large transverse momenta $10 \leq p_{t} \leq 25 \mathrm{GeV} / \mathrm{c}$. For the double $D^{0}$ production this contribution is not larger than $30 \%$ at $p_{t} \geq 5 \mathrm{GeV} / \mathrm{c}$ and $3 \leq y \leq 4.5$. These IC contributions for the single and double $D$-meson production [14] were obtained with the probability of the intrinsic charm taken to be $w_{c \bar{c}}=3.5 \%$ [11], and they will decrease by a factor of 3 when $w_{c \bar{c}} \simeq 1 \%$. Therefore, this value can be verified experimentally at the LHCb.

The presented predictions could stimulate measurement of the single and double D-meson production in $p p$ collisions at the CERN LHCb experiment in the kinematic region mentioned above to observe a possible signal for the intrinsic charm. The intrinsic beauty in the proton is suppressed by a factor of 10 , therefore its signal in the inclusive spectra of $B$-mesons will probably be very weak.

We also analyzed the inclusive $K^{-}$-meson production in $p p$ collision at the initial energy $E_{p}=158 \mathrm{GeV}$ and gave some predictions for the NA61 experiment going on at CERN, and made some predictions for the inclusive spectra of $K^{ \pm}$-mesons produced in $p p$ at the LHC energy $\sqrt{s}=$ $7 \mathrm{TeV}$. We showed that in the inclusive spectrum of $K^{-}$-mesons as a function of $p_{t}$ at the initial energy $E_{p}=158 \mathrm{GeV}$ and some values of their rapidities the signal of the intrinsic strangeness can be visible and reach about $200 \%$ and more at large momentum transfer we took. The signal of the intrinsic strangeness in the inclusive spectrum of $K^{-}$-mesons produced in $p p$ collision at $\sqrt{s}=7$ $\mathrm{TeV}$ can be about $200 \%$ and even $400 \%$ at $p_{t}>15 \mathrm{GeV} / \mathrm{c}, y=4.5$. The probability of the intrinsic strangeness to be about $2.5 \%$, as was found from the best description of the HERA and HERMES data on the DIS, see [15] and references therein.

\section{Acknowledgements}

We are very grateful to A.F Pikelner for his help with the MC calculations. We thank S.J.Brodsky, M. Gazdzicki, S.M. Pulawski and A.Rustamov for extremely helpful discussions and recommendations for the predictions on the search for the possible intrinsic heavy flavour components in $p p$ collisions at high energies. We are also grateful to V.A.Bednyakov, I.Belyaev, V.Gligorov, H.Jung, B.Kniehl, B.Z.Kopeliovich, A.Likhoded, P.Spradlin, M. Poghosyan, V.V Uzhinsky and N.P.Zotov for very helpful discussions. This work was supported in part by the Russian Foundation for Basic Research, grant No: 11-02-01538-a.

\section{References}

[1] J.W.Negele et al., Nucl.Phys. B [Proc.Suppl.] 128, (2004) 170; W.Schroers, Nucl. Phys. A 755 (2005) 333. 
[2] V.N. Gribov and L.N. Lipatov, Sov.J.Nucl.Phys. 15 (1972) 438; G. Altarelli and G. Parisi, Nucl.Phys. B 126 (1997) 298; Yu.L. Dokshitzer, Sov.Phys. JETP 46 (1977) 641.

[3] J.Pumplin, D.R.Stump, J.Huston, H.L.Lai, P.Nadolsky and W.K.Tung, J. High Energy Physics 07 (2002) 012; D.R.Stump, J.Huston. J.Pumplin, W.K.Tung, H.L.Lai, S.Kuhlmann and J.F.Owens, J. High Energy Physics 10 (2003) 046.

[4] R.S.T orne, A.d.Martin, W.G.Stirling, and R,G.Roberts, arXiv:04073 [hep-ph0].

[5] A.Aktas, etal., (H1 Collaboration), Eur.Phys. J.C40, (2005) 349; arXiv:0507081 [hep-ex].

[6] Brodsky S., Hoyer P., Peterson C., Sakai N., Phys.Lett. B 93 (1980) 451.

[7] Brodsky S., Peterson C., Sakai N., Phys.Rev. D, 23 (1981) 2745.

[8] J.F.Donoghue, E.Golowich, Phys.Rev.D 15 (1977) 3421.

[9] Pumplin J., Phys.Rev. D 73 (2006) 114015.

[10] Pumplin J., Lai H., Tung W., Phys.Rev. D 75 (2007) 054029.

[11] Nadolsky P. M., et. al., Phys. Rev. D 78 (2008) 013004.

[12] Polyakov M. V., Schafer A., Teryaev O. V., Phys.Rev. D 60 (1999) 051502.

[13] Goncalves V., Navarra F., Nucl.Phys. A 842 (2010) 59.

[14] G.I.Lykasov, V.A.Bednyakov, A.F.Pikelner and N.I.Zimin, Eur.Phys.Lett. 99 (2012) 21002; arXiv:1205.1131v2 [hep-ph].

[15] Jen-Chieh Peng, Wen-Chen Chang, Published in PoS QNP2012 (2012) 012 Plenary talk at Conference: C12-04-16; arXiv:1207.2193 [hep-ph].

[16] Litvine V. A., Likhoded A. K., Phys. Atom. Nucl. 62 (1999) 679.

[17] V.M.Abazov et al., Phys.Rev.Lett. 102 (2009) 192002; arXiv:0901.0739 [hep-ex].

[18] Vogt R., Prog. Part. Nucl. Phys. 45 (2000) S105.

[19] Navarra F. S., Nielsen M., Nunes C. A. A., Teixeira M., Phys.Rev. D 54 (1996) 842.

[20] Melnichouk W., Thomas A. W., Phys.Lett. B 414 (1997) 134.

[21] A.V.Efremov, Sov.J.Nucl.Phys., 19, 176 (1974).

[22] P.Nasson, S.Dawson \& R.K.Ellis, Nucl.Phys., B 303, 607 (1988).

[23] P.Nasson, S.Dawson \& R.K.Ellis, Nucl.Phys., B 327, 49 (1989).

[24] P.Nasson, S.Dawson \& R.K.Ellis, Nucl.Phys., B 3335, 260(E) (1989)

[25] R.D.Field,R.P.Feyman, Phys.Rev.D 15 (1977) 2590.

[26] R.P.Feyman, R.D.Field, and G.C.Fox, Nucl.Phys.B 128 (1977) 1.

[27] R.P.Feyman, R.D.Field, and G.C.Fox, Phys.Rev.D 18 (1977) 3320.

[28] M.L.Mangano, Physics-Uspekhi, 53 (2010) 109.

[29] S.Albino, B.A.Kniehl, G.Kramer (AKK08), Nucl.Phys. B803 (2008) 42.

[30] V.A. Bednyakov, A.A. Grinyuk, G.I. Lykasov, M. Poghosyan, Nucl.Phys. B 219-220 [Proc.Suppl.] (2011) 225; arXiv:11040532 [hep-ph].

[31] V.A. Bednyakov, A.A. Grinyuk, G.I. Lykasov, M. Poghosyan, Int.J.Mod.Phys. A27 (2012) 1250042.

[32] B. Kniehl, G. Kramer, I. Schienbein and H. Spiesberger, Eur.Phys.J., C72 (2012) 2082; arXiv:1202.0439v1 (2012) [hep-ph] . 